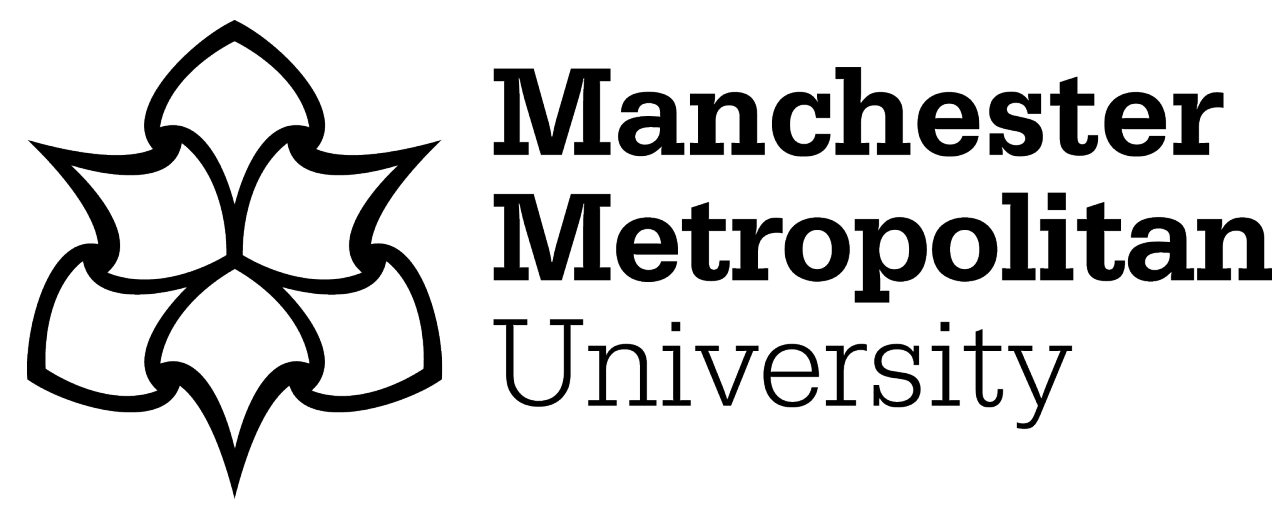

Amano, Kinjiro, Lou, Eric CW ORCID logoORCID: https://orcid.org/00000001-7099-1426 and Edwards, Rodger (2019) Integration of point cloud data and hyperspectral imaging as a data gathering methodology for refurbishment projects using Building Information Modelling (BIM). Journal of Facilities Management, 17 (1). pp. 57-75. ISSN 1472-5967

Downloaded from: https://e-space.mmu.ac.uk/620151/

Version: Accepted Version

Publisher: Emerald

DOI: https://doi.org/10.1108/JFM-11-2017-0064

Please cite the published version 


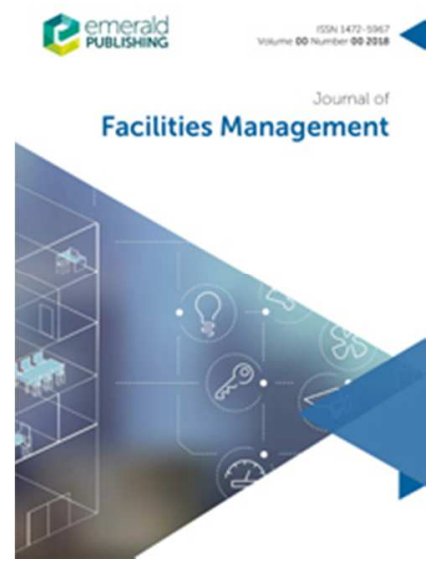

Integration of point cloud data and hyperspectral imaging as a data gathering methodology for refurbishment projects using Building Information Modelling (BIM)

\begin{tabular}{|r|l|}
\hline Journal: & Journal of Facilities Management \\
\hline Manuscript ID & JFM-11-2017-0064.R1 \\
\hline Manuscript Type: & Research Paper \\
\hline Keywords: & $\begin{array}{l}\text { BIM, hyperspectal imaging, laser scanning, point cloud, refurbishment, } \\
\text { information modelling }\end{array}$ \\
\hline
\end{tabular}




\section{Integration of point cloud data and hyperspectral imaging as a data gathering methodology for refurbishment projects using Building Information Modelling (BIM)}

\section{Purpose}

Building Information Modelling (BIM) is a digital representation of the physical and functional characteristics of a building. Its use offers a range of benefits in terms of achieving the efficient design, construction, operation and maintenance of buildings. Applying BIM at the outset of a new build project should be relatively easy. However, it is often problematic to apply BIM techniques to an existing building, for example as part of a refurbishment project or as a tool supporting the facilities management strategy, due to inadequacies in the previous management of the dataset that characterises the facility in question. These inadequacies may include information on as built geometry and materials of construction. By the application of automated retrospective data gathering for use in BIM, such problems should be largely overcome and significant benefits in terms of efficiency gains and cost savings should be achieved.

\section{Design/methodology/approach}

Laser scanning can be used to collect geometrical and spatial information in the form of a 3D point cloud and this technique is already used. However, since a point cloud representation does not contain any semantic information or geometrical context, such point cloud data must refer to external sources of data, such as building specification, construction materials, in order to be in used in BIM.

\section{Findings}

Hyperspectral imaging techniques can be applied in order to provide both spectral and spatial information of scenes as a set of high-resolution images. Integrating of a 3D point cloud into hyperspectral images would enable accurate identification and classification of surface materials and would also convert the 3D representation to BIM.

\section{Originality/value}

This integrated approach has been applied in other areas, for example in crop management. The transfer of this approach to facilities management and construction would improve the efficiency and automation of the data transition from building pathology to BIM. In this study, the technological feasibility and advantages of the integration of laser scanning and hyperspectral imaging (the latter not having previously been used in the construction context in its own right) is discussed and an example of the use of a new integration technique is presented, applied for the first time in the context of buildings.

Keywords: BIM, hyperspectral imaging, laser scanning, point cloud, refurbishment, facilities management 


\section{Introduction}

Building Information Modelling (BIM) is used to capture digitally a representation of the both functional and physical characteristics of a building or facility, thereby creating a knowledge sharing platform for all stakeholders involved in the life cycle of that building, from the clients and architects through to the team that carry out the final demolition, via the users of the building and its facilities management team. The use of BIM should therefore provide a far greater degree of integration between separate processes in the life cycle than was possible even in the recent past, for example, in the generation, review and management of CAD drawings, thermal performance simulation, regulatory compliance checking, drawing up of bills of quantities, materials procurement, project execution, maintenance and asset management. This integration should lead to the breaking down of the traditional partitions of the construction process, thereby delivering greater efficiency and hence time cost and savings.

The UK Government (HM Government, 2013) targeted as the key objectives of BIM implementation within the UK construction industry reduced costs, more rapid delivery of projects, reduced environmental impacts (including reductions in greenhouse gas emissions) and improvements in the competitiveness of exports to position the UK at the forefront of international construction. Within this document, the use of fully collaborative BIM was mandated for UK Government funded projects from 2016. Refurbishment is expected to constitute an large part of the workload in the global construction industry, driven to a great extent by the need to improvement the energy performance of existing buildings to as close a point as possible to that of new build buildings. In the UK, some 25 million dwellings, $(8.5$ million of which are in excess of 60 years old), contributing up to $27 \%$ of the total UK $\mathrm{CO}_{2}$ emissions and 1.8 million non-domestic properties (contributing 18\%) will require some level of refurbishment by 2050 in order for the UK to able to have any chance of meeting its treaty obligations to reduce $\mathrm{CO}_{2}$ emissions (Edwards and Townsend, 2011). As with many other business sectors, the construction industry will be forced to adopt digital approaches in the future. BIM is the way forward for design and long-term facility management. Its use provides a capability to handle increasing amounts of raw data and information, and indeed the achievement of the full potential of BIM will necessitate further improvements of this capability. Such improvements will be facilitated by access to such technological advances as the use of so called big data approaches (Hilbert and Lopez, 2011), virtual reality (Olbrich et al., 2013) and cloud computing (Beach et al., 2015). A BIM framework for use in the operation, maintenance and sustainability monitoring of existing buildings has already been described by McArthur (2015).

The implementation of BIM in a refurbishment project can be a major challenge due to the limited availability of accurate data about the original project. Data may have been badly managed in the past; for example, any available documentation may have been lost or damaged over the passage of time and the process of change management during the construction, operation and maintenance of the building may have been poor or non-existent (Gorse and Highfield, 2009). Dealing with the unrecorded use of hazardous materials such as asbestos during refurbishment, which was commonly used in commercial buildings in the 1960 s, would be a serious challenge that is often encountered in reality.

Availability of accurate spatial and geometric information of the facilities is critical for both the success of any refurbishment project and of maintenance strategies. Because of these needs, major benefits can be accrued by the application of modern technologies, such as laser scanning and digital imaging, Three-dimensional scanning of facilities has been implemented in BIM, for example by Goedert and Meadati (2008), and the demand for such scanning services has been increasingly growing. One of the key requirements for successful BIM is the automation of the information pipeline from data acquisition and its analysis, through to the storage of data. Accurate identification and classification of 
construction materials and fabrics is an essential requirement within in the automation process. It is in theory achievable through the use of several possible digital imaging techniques. In this paper, proposed methods of extracting semantic information from colour image and associated issues are discussed the integration of spectral information from the use of hyperspectral imaging with a 3D point cloud generated by laser scanning is considered. Bruno et al (2015) point out a number of knowledge gaps which are an impediment to the effective use of BIM in projects relating to historic buildings amongst which is the "Low level of development of effective hybrid systems made up of digital cameras, thermal imaging cameras and laser scanners for survey of complex buildings, beyond the inner spaces". They point out that their findings are in agreement with those of Ilter and Erger (2015). In neither paper is the use of spectral imaging mentioned amongst the possible options discussed.

In the present study, a novel approach for extracting semantic information is proposed for use in generating BIM data for existing facilities by integrating a 3D point cloud generated by laser scanning with hyperspectral imaging data, and the use of the approach is demonstrated for the first time.

\section{Functional requirements for BIM}

In order for use BIM to give satisfactory outcomes, there are several functional requirements that need to be taken into account. Accurate visualisation provided by generation of a 3D point cloud with laser scanning and associated image processing will facilitate the understanding of the spatial structure of facilities. This is essential to manage facility refurbishment and maintenance or retrofitting with BIM. For the generation of spatial information, detailed spatial information is however insufficient to enable the identification and classification of construction materials. This can be achieved by integrating extra information to the point cloud for example, planning records and documentations, colour images from external devices such as digital cameras captured at the same time as the 3D laser scanning and information from independent databases. Such additional information will improve the efficiency of using the model as well as the quality of the modelling itself.

In recent years, significant progress has been achieved towards automating detection and visualization of the as-built status of a project (Dimitrov and Golparvar-Fard, 2014; El-Omari and Moselhi, 2011; Han and Golparvar-Fard, 2015). The methods by these researchers include image-based sensing technologies and 3D remote sensing technologies. However, further improvements are still required in order to achieve more efficient and accurate measurement in practice. In the present study, the focus is set in visual information. Based on digital image information, the feasibility of efficient identification and classification of the building materials is addressed.

BIM enables users to access facility information more efficiently, effectively, and easily to share, edit, and reuse (Arayci, 2008; Ballesty et al., 2007; Howard and Björk, 2008). Consequently, facility proposals can be rigorously analysed, simulations can be performed quickly. Building performance can also be benchmarked, enabling improved and innovative solutions. BIM should hold not only information about individual objects in a facility but also information about geographical context and semantic meaning so as to facilitate analysis, simulation, and assessment (Arayci, 2008). While it is possible to generate a BIM model from a CAD-based model of a facility (as-designed condition), this model cannot capture detailed depictions of the state of a facility as it was actually built (as-built condition) or as it exists currently (as-is condition) (Barlish and Sullivan, 2012; Volk et al., 2014) unless there has been careful scrutiny of the final as-built and as-is final product. Documenting the asbuilt condition is more complex because the original information may be inaccurate or outdated if the management is poor. The information available through the as-built BIM 
depends largely on the state of the past documentation. Thus, the BIM protocol must consider usability of information and quality of the information management (Barlish and Sullivan, 2012; Solihin and Eastman, 2015).

Accurate visualisation of the 3D point cloud with advanced image processing will facilitate the understanding of the spatial structure of facilities. This is essential to manage facility refurbishment or retrofitting with BIM. For spatial information, laser scanning can capture the geometrical information as a form of point cloud. However, having only the spatial information is not sufficient to identify and classify construction materials. This can be achieved by integrating extra information to the point cloud; for example, planning records and documentations, colour images by external devices taken at the same time as the 3D laser scanning, and the information from independent database. Such additional information will improve the efficiency of using the model as well as the quality of the modelling itself.

In recent years, significant progress has been achieved towards automating detection and visualization of the as-built status of a project. These methods include image-based sensing technologies and 3D remote sensing technologies. However, further improvements are still required in order to achieve more efficient and accurate measurement in practice.

\section{Data acquisition}

\subsection{Laser scanning}

Laser scanning is the most commonly used 3D remote sensing technology in the built environment industry, as it is capable to address most requirements in generating a BIM for existing facility, such as accurate geometric data, its analysis, and monitoring the progress of the project (Zhu and Brilakis, 2009). Laser scanning assigns three dimensional spatial coordinates and intensity (reflectivity) at distinct points in a scene, which construct the data as a point cloud. This non-destructive data acquisition is useful in the imaging of existing facilities, in particular, historic and vulnerable buildings (Tang et al., 2010). The remote capture of cultural heritage buildings is valuable as an application of documentation and management of conservation (Mills et al., 2011). One of the unique features of point cloud is the ability to view the whole facility in a full degree of freedom, that is, from a variety of viewing angles. This feature enables users to interact flexibly the dense range point data in a virtual environment. Specially designed processing tools have been proposed to achieve automatic detection and identification of the building elements (Golparvar-Fard et al., 2009; Han and Golparvar-Fard, 2015; Tang et al., 2010). Laser scanning has been also used for construction quality control (Jaselskis, 2006), condition assessment, component tracking Teizer, 2015) and project progress monitoring (Golparvar-Fard et al., 2009).

A laser scanner achieves accuracy in the order of a few millimetres and the range of the measurement extends up to a few hundred metres (Fröhlich et al., 2000), but there are some variations in performance between different mechanisms (Boehler and Marbs, 2003). The accuracy of measurement is dependent on object specific parameters such as location of the object, surface reflectivity, surface texture, specific surface materials, and imaging environment. The variable imaging condition encountered in in the natural environment, for example sunlight and reflections, also affects the quality of the scanning. There are various challenges involved in data acquisition by laser scanning and its implementation to BIM (Kiziltas et al., 2008). The range of the laser signal imposes physical limits. The power of the laser signal defines the effective distance of the objects (Boehler and Marbs, 2003). In addition, a facility with large glass windows cannot be accurately captured because the laser beams pass through the window glass (Zhang, 2000). Analogous to the other sensing devices, any objects occluded in line-of-sight, or any moving objects will not be captured. These problems can be solved by multiple scans at different locations. This requires careful planning of the scanning process in advance of data acquisition. In order to maintain the 
accuracy of scanning, specific sensor calibration is necessary as a matter of routine (Boehler and Marbs, 2003). As described earlier, the point cloud data only contains Cartesian coordinates and intensity values at every point, and does not carry any semantic information. Working with such featureless data to explore geometric reasoning is generally tedious, time consuming, and often causes errors (Kiziltas et al., 2008). Implementations of the semantic information, context of the scene from external information sources (such as imaging devices) and information processing are suggested in order to overcome these disadvantages (Jung et al., 2014). Significant challenges in the process of processing 3D point cloud into a usable form for use in BIM are the extraction of reliable and meaningful data from the raw data, the integration of external information, and the automation of these processes.

\subsection{Point cloud processing}

The generation of the as-built BIM with the 3D point cloud can be divided into three main steps (Jung et al., 2009; Tang et al., 2010). Firstly is data acquisition, where dense point measurements at key locations throughout the facility are performed by the laser scanners; secondly comes data processing, where the sets of point measurements are filtered to remove noise and artefacts to from a set of point cloud data, and thirdly functional processing and implementation to BIM, where the low-level point cloud or surface representation is transformed into a semantically rich BIM, involving identification and classification of objects and components in the facility. The second and third processes may require a large amount of computationally intensive activity, depending on the project. The pipeline of the data processing can be illustrated as in Figure 1.

Figure 1. Protocol of point cloud processing in a flow chart.

Raw point cloud data can include so called outlier data points caused by noise in ambient environment and sensors. Such outliers and noise can be removed computationally. Scanning can be often performed at multiple locations to overcome problems such as occlusion of objects. The set of multi sourced scan data need to be registered to compile to one dataset, where any duplications will be removed. Depending on the requirements and levels of details required, the point cloud could be subsampled to reduce the computational intensity of the data processing. These steps are fundamental to achieve better results in further processing, such as smoothing, normal estimation and (optionally) surface reconstruction. It is necessary to distinguish different states of a material, for example, concrete in formwork or finishes, and different materials, for example, masonry units or facade bricks, during the 3D modelling process (Dimitrov and Golparvar-Fard, 2014) and to reconstruct the surfaces so that its structure and scene contents can be identified and implemented to the BIM of the facility.

\subsection{Image registration}

The main objective of the image registration is to align individual point clouds and fuse them to a single point cloud so that subsequent processing, such as segmentation and reconstruction of surfaces, can be performed efficiently. The registration finds the relative pose (position and orientation) between views in a global coordinate frame, such that the overlapping areas between the point clouds match as well as possible. The well-established open source library, for example, Point Cloud Library (Rusu and Cousins, 2011; Holz et al., 2015) or the software provided by the scanner manufacture in question can be used. The successful performance of registration relies on finding correspondences between the points and estimating a transformation matrix. When more information is available, for example, colour information or information about a local surface normal or curvature the points, the accuracy of the registration can be improved. A fundamental problem of registration is that 
these correspondences are usually unknown and need to be determined by the registration algorithm itself.

The registration of multiple point clouds can be split into the following steps (Rusinkiewicz and Levoy, 2001). Selection, the sampling of the input point clouds; matching, the estimation of the correspondences between the points in the subsampled point clouds; rejection, the filtering of the correspondences to reduce the number of outliers and finally alignment, assigning an error metric and minimizing it to find the optimal transformation. Two major classes of registration algorithms are the iterative registration algorithms (ICP) and feature-based registration algorithms. In the ICP algorithm (Besl and McKay, 1992), no feature descriptors are computed, but instead closest points in Cartesian space are considered to correspond to one another. A transformation is estimated that minimizes the Euclidean distances between found pairs of closest points in the least squares sense. The process of determining corresponding points in the two data sets and computing the transformation that aligns them is iteratively repeated until either convergence occurs or else until another termination criterion is reached.

For the iterative approach, a basic method is to scan through the whole data, point by point. There are various data structures available for the performance of rapid searches, such as octrees and kd-trees. An alternative approach would be to use the projective nature of the depth images with a reasonable approximation. Each point in the cloud corresponds to a pixel in the depth image, allowing projections from source points in world coordinates (representation of a position in physical world) to the camera plane of the target frame. This method is faster and simpler than kd-trees, but camera calibration (Heikkila and Silven, 1997; Zhang, 2000) has to be performed in order to obtain the intrinsic and extrinsic camera parameters. The point correspondences are examined by various algorithms such as RANdom SAmple Consensus (RANSAC) (Fischler and Bolles, 1981) and normal compatibility. Alignment is then performed with the rigid transformation composed of rotations and translations. The computation of solving the rigid transformation that minimizes the error of the point pairs is to be repeated (iterative registration) until the error becomes smaller than criteria.

The feature-based registration approach can be fast and automatic, determining point-topoint correspondences between 3D key points extracted from both point clouds via matching the associated key point descriptors. Another possible algorithm is Normal Aligned Radial Feature (NARF) (Steder et al., 2010), a method specifically designed to extract salient key points in range images. It focuses on detecting points along object depth borders (Tombari et al., 2012). For each detected key point, a local descriptor (a compact representation of a point's local neighbourhood) is determined. In contrast to global descriptors that are computed to determine correspondences between the two point clouds and describe a complete object or point cloud, local descriptors try to resemble shape and appearance only in a local neighbourhood around a point, and are suitable for representing it in terms of matching. For example, the Fast Point Feature Histogram (FPFH) descriptor (Rusu et al., 2009) stores the relative orientation of normals and distances between point pairs falling within the spherical neighbourhood of a key point. Point pairs are formed by the key point and its nearest neighbours, as well as by the nearest neighbours of each key point's neighbour. The performance of the registration could be improved by inserting objects (to be the image features) physically and manually into the scene. These are usually either spheres covered with uniform white or else plates of a chequered pattern). The reference objects can be used for both geometrical and spectral calibration if their surfaces can be painted with a spectrally neutral pigment (for example, barium sulphate samples), it would be useful to perform spectral calibration. New laser scanners have a built-in RGB camera. The colour information is integrated into point cloud to assist object recognition or visual inspection. A number of the computational approaches to achieve the integration of point cloud with 
external sensors and imaging devices have been proposed (Dimitrov and Golparvar-Fard, 2014; Fathi and Brilikas, 2011; Golparvar-Fard et al., 2011).

\subsection{Advanced imaging: colour information for surface recognition}

Three main research challenges in 3D point cloud processing are segmentation based on geometric appearance, object recognition in the scene, and inter-object relationships. Recently proposed methods are the visual appearance approach and the computational approach (Han and Golparvar-Fard, 2015). In order to identify and classify the objects in the facilities or scenes, it is necessary to attach additional semantic information to the point cloud (Golparvar-Fard et al., 2011; Yalcinkaya and Singh, 2015). Colour images produced by a conventional digital camera are a favoured documentation medium, because they contain rich information about geometry and appearance and in addition images can be collected quickly and inexpensively. Computer vision and image processing techniques on the image data provide useful information about the objects in the scene and facilities, so as to aid to infer scene context, object properties, and its semantic meaning. Site photographs, for example, allow capture of dynamic events on construction sites such as moving operatives and equipment at high update rates which can be complementary to the detailed and static range data on the fixed objects collected by laser scanners. The current status of remote sensing technology suggests that technologies should be combined to establish asbuilt models, because no single remote sensing method alone can solve all the requirements of industrial users (El-Omari and Moselhi, 2008). This logic is applicable to the extraction of the present proposition.

The semantic information or context of the scene can be extracted through independent imaging, pre-examined data, or post image processing (Jung et al., 2014; Jang et al., 2015). A number of computational algorithms have been reported to provide better solutions for this approach. For instance, Golparvar-Fard et al. (2011) presented a new algorithm based on Structure-from-Motion (SfM) coupled with Multi-View Stereo and Voxel Colouring for generating dense 3D point cloud models from unordered imagery. Several research projects such as Golparvar-Fard et al. (2011), Klein et al. (2012) and Zhu and Brilakis (2009) have also focused on evaluating the accuracy of as-built 3D modelling from photos and laser scanners. Fathi et al. (2011) generated 3D point cloud models of infrastructure using video streams. Recent works by Son and Kim (2012) proposed using colour to identify concrete elements and construction equipment. Dimitov and Golparvar-Fard (2014) proposed the image-based material recognition algorithm based on a statistical distribution of filter (linear Gaussian derivative filter outputs) responses over the images, in form of primitives such as edges, spots, waves, and Hue-Saturation-Value (HSV) colour values. The statistical distribution of filter responses has been shown to be a good descriptor for texture recognition (Leung and Malik, 2001). They used a so-called "Bag of Words" pipeline (a technique used in natural language processing and information retrieval) for forming statistical distributions of both filter responses and HSV colour values, and a multiple binary SVM classifier that can robustly learn and infer construction material categories. They established a robust material classification method for semantically rich as-built 3D modelling and construction monitoring purposes. Using their own in-house Construction Materials Library, an average classification rate was over $97 \%$. Image synthesis techniques produced accurate results for images as small as $30 \times 30$ pixels in size. Leung and Malik (2001) suggested that further testing various types of features and the classification algorithm would be needed in order to be sure of the robustness and high level of accuracy of the technique in uncontrolled construction environments. Han and Golparvar-Fard (2015) proposed a manual integration of $3 \mathrm{D}$ point cloud and 2D image patches to facilitate 4D BIM. The appearance-based material classification method was aimed to monitor construction progress deviations. The method leverages 4D BIM and 3D point cloud models generated from site photo-logs using $\mathrm{SfM}$ techniques. The algorithm uses the back projection of the $2 \mathrm{D}$ colour imagines so that the occluded objects and elements can be seen, the 2D patches are sampled per element and are convolved with texture and colour filters and their concatenated vector-quantized 
responses are compared with multiple discriminative material classification models that are relevant to the expected progress of that element. With an extended version of the Construction Material Library for training or testing the material classifiers achieve an average accuracy over $92 \%$. This algorithm may be limited to the dependency on comprehensive construction materials library (Jang et al., 2015).

Colour information is useful to identify and distinguish materials, but it has been noted that the accuracy of the images often suffer as a consequence of limitations in the conventional RGB camera (Son and Kim, 2012). The accuracy of colour rendering depends on the environmental condition where the image is being acquired and characteristics of the imaging sensors. The colour appearance of the surfaces, for example, varies with the scene illumination and this is an issue even if the RGB values are converted to the other colour spaces. Figure 2 shows an image of a piece of office furniture acquired by the built in RGB camera built into the laser scanner compared with an image of the same piece of furniture taken at the same time that has been acquired by a standalone RGB camera Significant differences between the colour rendering appearance obtained by the two cameras can be seen. Differences are not confined to full colour applications. For example, Dimitrov and Golparvar-Fard (2014) report difficulties in assessing the state of concrete based only on colours, as concrete invariably appear as grey in images. More fundamentally, due to the limited number of the channels (sensors) over the colour spectrum in a RGB camera, these imaging devices cannot always produce correct colour appearance of surfaces with complex spectral properties, such as metameric surfaces (Foster et al., 2006). Characteristics of materials can be represented by the spectral profile of the surfaces. The estimated spectral reflectance values are physical properties of the material itself and are independent of scene illumination. It would be therefore useful if the spectral information could be integrated into the 3D geometrical information so that the point cloud can be meaningfully recorded in BIM. Spectral characteristics of urban construction materials, such as concrete and clay tiles exhibiting the effects of aging, have been examined in order to establish a spectral library so as to be implemented in BIM (Nasrudin and Shafri, 2011). The spectral characteristics of asphalt road aging and deterioration have been studied in detail by Herold and Roberts (2005).

Figure 2. Examples of misrepresentation of colour appearance by a RGB camera.

\section{Spectral imaging}

\subsection{Hyperspectral imaging}

The colour and reflectivity of surfaces, formally defined as spectral reflectance, provide more precise indications of the material composition of the object than colours themselves. This is not only because colour appearance in an image depends on how the image was acquired, but also because different materials absorb and reflect the impinging light in a wavelengthdependent manner. Thus, the examination of materials and their classification can not only be assessed visually but also spectrally.

Hyperspectral imaging systems have evolved to include not just three colour channels covering the visible spectrum, but over many channels, perhaps as many as several hundreds, encompassing the visible the near-infrared (NIR) shortwave infrared (SWIR) components of the electromagnetic spectrum (Foster et al., 2006; Coffey, 2015). The thin slices of the images over spectral range gave more detailed information than the commercial RGB camera and human eye. This imaging method is aimed mainly to exploit the materials comprising the various objects in a scene where lights reflect, scatter, and being absorbed, depending on characteristic of material. If the radiation arriving at the imaging sensor is measured at a number of wavelengths, over a sufficiently broad spectral band, the resulting 
spectral signature can be used to identify the materials in a scene and discriminate among different classes of material. Each pixel in the hyperspectral image holds spectral information. The demand for hyperspectral imaging has grown significantly in the recent years because of the expansion of research applications, for example, in agriculture (Behmann et al., 2015) and cultural heritage (Granero-Montagud et al., 2013). Hyperspectral imaging is currently limited to two dimensions and requires specific spectral calibration at each image acquisition. The reflected light, spectral radiance $L s(\lambda)$, that a sensor records is the product of the impinging scene radiance $L i(\lambda)$ and the material reflectance spectrum $R(\lambda)$, as a function of wavelength $\lambda$ :

$$
\operatorname{Ls}(\lambda)=R(\lambda) \times L i(\lambda)
$$

If the illumination spectrum is known, the material reflectance spectrum can be recovered from the observed spectral radiance over those regions of the spectrum and this information that allows materials to be identified. The more wavelengths at which the spectral radiation from surfaces can be determined, the more information about the materials in a scene can be provided. As with laser scanning, hyperspectral imaging is a non-destructive technique.

\subsection{Hyperspectral imaging systems}

The principle of hyperspectral imaging and the system structures can be represented by forming a three dimensional hyperspectral cube with the two dimensions of the horizontal and vertical axes on an image slice and wavelength, as shown in Figure 3(a). There are three different types of hyperspectral imaging systems available, each of which can be explained in terms of how the hyperspectral cube is divided: firstly, the spectral scan system Figure 3 (b) based on, a tuneable filter with monochromatic digital camera (Fichler and Bolles, 1981; Hardeberg et al., 2002); secondly, a line-scan system Figure 3(c) based on an optical slit or gratings which spread up the incoming light of a line into its spectral composition (Gilden Photonics, 2015); thirdly, a snap shot method based on, a set of spectral filters built-in on-chip (Imec, 2015) (not shown in Figure 3). The first system, the spectral scanning system, is the type used in production of the example image shown in Section 5 of this paper. Both spectral and spatial calibrations are required in the system (lens and sensors) to achieve the accuracy. Precision of the spectral profile contributes to performance in object and material recognition and classification. However, the quality of hyperspectral imaging data depends strongly on the measuring setup. The arrangement of the sensor to the object and the light source has to be considered most carefully, in particular, when outdoor imaging is being conducted. This is most important within the context of BIM, as an appreciable proportion of data acquisition will take place outdoors.

Figure 3. Schematic diagram explaining the classification of hyperspectral imaging systems.

\subsection{Post image processing: estimation of surface reflectances}

Data acquisition with hyperspectral imaging takes more time than conventional RGB cameras due to the number of the channels used and also due to differences in the mechanisms used for the production of images. The exposure time on each wavelength is pre-defined so that the intensity on pixels can reach certain threshold values defined by users. With the spectral scanning system, three different types of images are to be acquired: scene data, illumination data, and dark noise data of the imaging device. Scene data acquisition should be performed with the reference surfaces in a scene for the spectral calibration purpose. The spectral characteristics of these reference surfaces needed to be verified externally. The illumination data can be acquired by placing a neutral uniform field in front of the target scene in line of the camera so that the spatial distribution, uniformity, over the image plane can be captured. The dark noise is the response of the system without any input signal. Removing the spatial non-uniformity of the illumination and noise from the 
scene data, effective spectra on each pixel are computed, and then by normalising the reference spectrum by the intensity of the reference area, spectral reflectances are estimated.

\subsection{Limitations of hyperspectral imaging}

As with laser scanning, hyperspectral imaging does work well on the regions of specular reflection, strong mutual reflection, or of shadow in a scene. Hyperspectral imaging takes more acquisition time than conventional RGB camera and its use requires spatial and spectral calibrations in order to achieve sufficient accuracy. Information about scene illumination, intensity and direction at the light source should be recorded at the time of the image acquisition. The calibration is in general performed by inserting reference calibration markers in the scene, and the measurement with spectrometer would be associated. Hyperspectral imaging technique is adversely affected by changes in natural environment such as movement of objects in the scene and varying lighting conditions. The intensity of light may also vary as a function of the distance from the light source, viewing camera angles, aperture of the lens, and exposure time. These depend mostly on the system setup and imaging environment. The spectral range needs to be identified depending on the target materials in the scene or facilities, because the spectral characteristics of construction materials have large variation across the spectral range (Herold and Roberts, 2005; Nasarudin and Shafri, 2011).

\section{Image integration}

Having gained the spectral identity, spectral characteristics can be correlated to the spatial or geographical correlation to the point cloud. That is, the integration of the spectral data to the point cloud can achieve accurate material identification and classification, and will be more robust than the conventional colour image in RGB. One of the unique features of using the $3 \mathrm{D}$ point cloud data is the ability to manipulate and view data in a full degree of freedom, from a variety of viewing angle, camera positions, and viewing distance. Users can interact and manipulate the dense range point data flexibly, allowing for construction of as-built conditions in a virtual environment (Jaselskis et al., 2003). With this flexible feature, it is possible to adjust the viewing angle to a certain degree so that the projection of the 3D image can closely correspond to the 2D image. Once the 3D point cloud is projected on to the same image dimension (2D), the integration will be performed by applying the image registration. The image registration is run by detecting correspondences of key features between the images, for example, intensity contrast, gradients, edge, and surface normal, as previously addressed in the section above. A range of the existing image processing algorithm in 2D digital images is applicable to each of the hyperspectral images (each wavelength). This will enable to extract further details of the material information, such as edge detection, across spectra. To improve the quality of high dimensional data, the measurement specifications have to be considered in detail, since spectral information of surface materials could be influenced by geometric configuration of sensor, illumination, and the scene geometry. For example, the analysis may be performed on the limited wavelength of the dominant spectra depending on the scene (for example, the middle of visible spectra for the scene of green foliage). Integration of the imaging data will provide more robust and collect essential information for BIM. The integrated data between the 3D point cloud and the 2D hyperspectral image can deliver both geometric and spectral information. Such information improves the quality for tasks such as surface segmentation, classification, detection and assessment of the surface material conditions.

The integration will be based on the image registration with image feature detection. There are several algorithms to detect the key features: Binary Robust Invariant Scalable Keypoints (BRISK) (Leutenegger et al., 2011); Speeded-Up Robust Features (SURF) (Bay et al., 2008); Maximally Stable Extremal Regions (MSER) (Matas et al., 2002) and Fast 
Retina Keypoint (FREAK) (Alahi et al., 2012). The combination of these features is also applicable. Instead of the computational key features, it is possible to use reference objects or markers physically placed in the scenes. These image registration algorithms are applied to the registration of $3 \mathrm{D}$ point cloud as well as the 2D images (Rusu et al., 2008). The detection of any key features depends on the image contents. If the scene consists of, for example, a large uniform surface or walls, it is difficult to detect any key features. In such circumstances alternative registration algorithms could be considered (Behmann et al., 2015; Granero-Montagud et al., 2013; Tamas and Kato, 2013), or the users can physically provide specific reference objects at the time of data acquisition.

The integration between 3D point cloud and spectral images have previously been reported in other research disciplines, for example, the characterisation of plants and plant disease detection (Behmann et al., 2015; Shultz et al., 2001) and examination of art objects and other cultural heritage items (Granero-Montagud et al., 2013) used an integrated imaging system consisting of two different optical techniques: a hyperspectral imaging with electronically tuneable filters and 3D scanning, using structured light projection. Together with multi-sensor data merging and information processing, estimates of artwork deterioration and degradation were made. The integration (fusion) of the 3D point cloud to colour RGB images has been reported by Tamas and Kato (2013) together with the use of a new camera calibration method. As Behmann et al. (2015) demonstrated in the field of plant disease detection, data fusion between 2D hyperspectral data and the 3D geometrical data is achieved by the use of a transformation matrix. First, the parameters of the corresponding $3 \mathrm{D}$ ray for each pixel of the hyperspectral image are calculated. The observed surface point of the plant can then be determined. The calculation of the $3 D$ rays is performed by $a$ camera calibration procedure expressed by a semi homogenous projection matrix. For this method, the mathematical determination of the camera model and accurate calibration procedure are particularly important. The use of a reference object delivers automatically detectable surface points with known internal coordinates, which defines a unique coordinate reference frame, and achieves more accurate calculation.

\section{Demonstration of the new integration process}

The principles of the proposed new integration process are best illustrated by the use of real scans. Figure 4 represents an example of the image registration by means of the integration of images produced by the use of two different 2D imaging systems, taken by the different imaging system from different viewing distance and angles, shown in Fig. 4 (a) and (b). Within each of the images (a) and (b), a small sub-region has been selected, where a common reference object of a relatively distinctive nature is located. (in this example, the three lamps on the lamp-post on the bottom of the image). Between the two sub-regions, the normalised cross-correlation has been used to find the transformation so that the two images are overlapped. Next, image registration using the SURF algorithm was performed to make further alignment between the two images, the transformed image (a) and (b). On Fig. 4(c), the transformed image (a) is overlapped on the image (b), coloured in blue and red, respectively, for visibility. The locations of the image key features on Fig.4 (a) (after transformation) and (b) are shown by crosses and open circles, respectively. The corresponding features were estimated and a transformation matrix generated. The final result after another image transformation based on the key features is shown on Fig. 4 (d), where the two images are sufficiently registered. The quality of the image registration is controlled by adjusting several optimisation parameters, such as the number of iterations, step size, and tolerance level. When the image integration is completed successfully, the spectral properties extracted at each pixel can be applied to the point cloud data.

Figure 4. An demonstration image registration. 


\section{Issues with integration}

The proposed method of integration between 3D point cloud and hyperspectral imaging will improve the efficiency and accuracy of BIM for existing buildings. However, certain disadvantages would have to be addressed during the further development of the method. The projection to $2 \mathrm{D}$ loses the advantage of having $3 \mathrm{D}$ information, that is, depth and occlusion. Any of the image information on the invisible surfaces from a viewing angle will be no longer available. This will be problematic if any significance or damage may exist on those surfaces. The 3D information provides useful information about the effect of scene illumination on the facilities. For example, changes of shadow and shade in direction and area along with daylight changes by simulation will be useful information in designing and retrofitting buildings and facilities. Because spectral data is being processed, the effects of chromatic aberration have to be considered. The fact that each spectral channel has different focus points causes what is referred to as "colour fringe" at the edge of surfaces in the image. The correction of this chromatic aberration is important because edges of surfaces are essential clues in their identification and classification. The correction is performed during the post processing stage (for example, the image registration across the spectral channels) or using specially designed optical devices (for example, an achromatising lens) in front of the sensor.

Apart from the proposed image integration method, the direct image fusion between 3D point cloud and 2D colour image has been reported by Tamas and Kato (2013) who pointed out the particular importance of characterisation of the imaging devices, not only of the sensor's spectral and spatial sensitivities, but also optical calibration of the imaging systems is required in order to achieve high accuracy in image fusion. Zhang (2000) considered camera calibration is as a necessary step in $3 \mathrm{D}$ computer vision to extract metric information from $2 \mathrm{D}$ images. Without reliable knowledge of the optical properties of the lenses of the cameras used, errors will be hard to avoid. Camera calibration is the process of determining both intrinsic and extrinsic parameters. Intrinsic parameters relate to the camera's internal characteristics, such as its focal length, skew, distortion, and image centre, whilst extrinsic parameters describe its position and orientation in the world. Knowing these intrinsic parameters is an essential first step for 3D computer vision, as it allows users to estimate the scene's structure in Euclidean space and removes lens distortion, which improves accuracy (Darrodi et al., 2015). Such characterisation of the devices should be performed ahead of use of the system.

Hyperspectral 3D models provide a large amount of high-dimensional data that require advanced data analysis methods. The complexity and computational cost of the analysis is much higher than that in a single image property. It is possible to reduce such demands by subsampling the data. However, the requirements in the final results in terms of both spectral and spatial resolution should be considered in advance. Difference in spatial resolution of the imaging systems may cause inaccuracy in image registration. Spectral characteristics of urban materials, such as, concrete and clay tiles, have been used to establish a spectral library for BIM (Nasrudin and Shafri, 2011). These data should be taken into account to define the spectral range of hyperspectral imaging. A much bigger spectral database for use as library in BIM will be needed in order to underpin the widespread usage of hyperspectral imaging. This will involve the standardisation of data formats.

\section{Conclusions}

This paper has considered the implementation of the digital spatial and spectral data acquisitions into BIM. It proposes the development of a new method for the integration of a $3 \mathrm{D}$ point cloud the image and a 2D hyperspectral image. The proposed method has been demonstrated to work achieved under controlled conditions. In order to progress from this 
proof of concept position to a usable tool for use with BIM, a number of technical issues would have to be addressed. Some of these issues could be resolved by careful preplanning of the imaging protocol for larger applications, but the reduction of computational costs for the integration of point cloud and hyperspectral data will require the development of better intelligent processing algorithms.

By achieving the ability to achieve high quality image integration, the identification and classification of construction materials will be more efficient. This will greatly improve the usability of BIM in refurbishment projects involving existing facilities, resulting in greater efficiency, reliability and reduced costs.

Refurbishment and retrofit projects aimed at the reduction of carbon emissions will form a greatly increased proportion of construction activity work in the UK. The achievement of agreed carbon emission targets is an imperative and is the single greatest environmental challenge of the day. This means that there are powerful environmental and commercial drivers for the increased use of BIM in refurbishment projects and also for continued development and improvement of data gathering and processing tools. The facilities management sector will accrue efficiency and cost benefits by having access to a technique which allows the integration of dimensional data collection with materials characterization

\section{Acknowledgements}

This research is supported by the Newton-Ungku Omar Institutional Links Fund (British Council) [172726659]. Preliminary report of the work was presented at the 4th International Building Control Conference, 07 March 2016. We thank D. H. Foster and S. M. C. Nascimento for the use of a set of hyperspectral images of natural scenes and image samples. 


\section{References}

Alahi, A., Ortiz, R. and Vandergheynst, P. (2012), "FREAK: Fast Retina Keypoint", Proceedings in Computer Vision and Pattern Recognition (CVPR), 510-517.

Arayici, Y. (2008), "Towards building information modelling for existing structures", Structural Survey, 26(3), 210-222.

Ballesty, S., Mitchell, J ., Drogemuller, R., Schevers, H., Linning, C.S., Singh, G. and Marchant, D. (2007), Adopting BIM for Facilities Adopting BIM for Facilities Management: Solutions for Managing the Sydney Opera House, Cooperative Research Centre for Construction Innovation (CRC), Queensland, Australia.

Barlish, K. and Sullivan, K. (2012), "How to measure the benefits of BIM - A case study approach", Automation in Construction, 24, 149-159.

Bay, H., Ess, A., Tuytelaars, T. and Van Gool, L. (2008), "Speeded-Up Robust Features (SURF)", Computer Vision and Image Understanding, 110(3), 346-359.

Beach, T., Rana, O., Rezgui, Y. and Parashar, M. (2015), "Governance Model for Cloud Computing in Building Information Management", IEEE Transactions on Services Computing, 8(2), 314-327.

Behmann, J., Mahlein, A.K., Paulus, S., Kuhlmann, H., Oerke, E.C. and Plümer, L. (2014), "Generation and Application of Hyperspectral 3D Plant Models", in Agapito, L., Bronstein, M.M. and Rother, C. (Eds.), Computer Vision - ECCV 2014 Workshops, Springer, 8928, 117-130.

Besl, P.J. and McKay, N.D. (1992), "A method for registration of 3-D shapes, Pattern Analysis and Machine Intelligence", IEEE Transactions, 14(2), 239-256.

Boehler, W. and Marbs, A. (2003), Investigating Laser Scanner Accuracy, i3mainz, Institute for Spatial Information and Surveying Technology, University of Applied Sciences, FH Mainz, Germany.

Bruno, S., De Fino M. and Fatiguso, F. (2015), "Historic Building Information Modelling: performance assessment for diagnosis-aided information modelling and management", Automation in Construction, 86, 256-276.

Darrodi, M.M., Finlayson, G., Goodman, T. and Mackiewicz, M. (2015), "Reference data set for camera spectral sensitivity estimation", Journal of the Optical Society of America A: Optics, Image Science and Vision, 32(3), 381-391.

Dimitrov, A. and Golparvar-Fard, M. (2014), "Vision-based material recognition for automated monitoring of construction progress and generating building information modeling from unordered site image collections", Advanced Engineering Informatics, 28(1), 37-49.

Edwards, J. and Townsend, A. (2011), Buildings under refurbishment and retrofit, Carbon Action 2050 White papers, Chartered Institute of Building (CIOB), England.

El-Omari, S, and Moselhi, O. (2008), "Integrating 3D laser scanning and photogrammetry for progress measurement of construction work", Automation in Construction, 18(1), 1-9.

El-Omari, S, and Moselhi, O. (2011), "Integrating automated data acquisition technologies for progress reporting of construction projects", Automation in Construction, 20(6), 699-705.

Fathi, H. and Brilakis, I. (2011), "Automated sparse 3D point cloud generation of infrastructure using its distinctive visual features”, Advanced Engineering Informatics, 25(4), 760-770.

Fischler, M.A. and Bolles, R.C. (1981), "Random sample consensus: a paradigm for model fitting with applications to image analysis and automated cartography", Communication ACM, 24(6), 381-395.

Foster, D.H., Amano, K., Nascimento, S.M.C. and Foster, M.J. (2006), "Frequency of metamerism in natural scenes", Journal of the Optical Society of America A: Optics, Image Science and Vision, 23(10), 2359-2372.

Fröhlich, C., Mettenleiter, M., Härtl, F., Dalton, G. and Hines, D. (2000), Imaging laser radar for 3-D modelling of real world environments, Sensor Review, 20(4), 273-282.

Gilden (2015), Hyperspectral Imaging, Gilden Photonics Ltd, Glasgow, Scotland.

Goedert, J D. and Meadati, P (2008), "Integrating construction process documentation into building information modelling", Journal of Construction Engineering and Management, 134(7).

Golparvar-Fard, M., Bohn, J., Teizer, J., Savarese, S. and Peña-Mora, F. (2011), "Evaluation of image-based modeling and laser scanning accuracy for emerging automated performance monitoring techniques", Automation in Construction, 20(8), 1143-1155.

Golparvar-Fard, M., Peña-Mora, F. and Savarese, S. (2009), "Application of D4AR - A 4-Dimensional augmented reality model for automating construction progress monitoring data collection, processing and communication", ITcon, 14, 129-153

Gorse, C. and Highfield, D. (2009), Refurbishment and Upgrading of Buildings, 2nd edition ed., Spon Press, Abingdon, England. 
Granero-Montagud, L., Portalés, C., Pastor-Carbonell, B., Ribes-Gómez, E., Gutiérrez-Lucas, A., Tornari, V., Papadakis, V., Groves, R.M., Sirmacek, B., Bonazza, A., Ozga, I., Vermeiren, J., van der Zanden, K., Föster, M., Aswendt, P., Borreman, A., Ward, J.D., Cardoso, A., Aguiar, L., Alves, F., Ropret, P., Luzón-Nogué, J.M. and Dietz, C. (2013), "SYDDARTA: new methodology for digitization of deterioration estimation in paintings, Proc. SPIE 8790, Optics for Arts, Architecture, and Archaeology IV, 8790.

Han, K.K. and Golparvar-Fard, M. (2015), "Appearance-based material classification for monitoring of operation-level construction progress using 4D BIM and site photologs", Automation in Construction, 53, 44-57.

Hardeberg, J.Y., Schmitt, F. and Brettel, H. (2002), "Multispectral color image capture using a liquid crystal tunable filter", Optical Engineering, 41(10), 2532-2548.

Heikkila, J. and Silven, O. (1997), A four-step camera calibration procedure with implicit image correction, Proceedings on Computer Vision and Pattern Recognition, 1106-1112.

Herold, M. and Roberts, D. (2005), "Spectral characteristics of asphalt road aging and deterioration: implications for remote-sensing applications", Applied Optics, 44(20), 4327-4334.

Hilbert, M. and López, P. (2011) "The World's Technological Capacity to Store, Communicate, and Compute Information", Science, 332(6025), 60-65.

HM Government (2013), Construction 2025: Industrial Strategy for Construction, Department for Business, URN BIS/13/955, London, England.

Holz, D., Ichim, A., Tombari, F., Rusu, R. and Behnke, S. (2015), "Registration with the Point Cloud Library: A Modular Framework for Aligning in 3-D”, Robotics \& Automation Magazine, 22(4), 110124.

Howard, R. and Björk, B.C. (2008), “Building information modelling - Experts' views on standardisation and industry deployment", Advanced Engineering Informatics, 22(2), 271-280.

Ilter and Erger (2015). D. Ilter, E. Ergen. "BIM for building refurbishment and maintenance: current status and research directions", Structural Survey, 33 (2015), 228-256.

Imec (2015), Hyperspectral imaging at Imec, http://www2.imec.be/be en/research/image-sensorsand-vision-systems/hyperspectral-imaging.html (Accessed 15 March 2015).

Jang, J., Park, M.W., Vela, P.A. and Golparvar-Fard, M. (2015), "Construction performance monitoring via still images, time-lapse photos, and video streams: Now, tomorrow, and the future", Advanced Engineering Informatics, 29(2), 211-224.

Jaselskis, E.J. (2006), Using Scanning Lasers for Real-Time Pavement Thickness Measurement, Phase I (TR-538), lowa State University Institute for Transportation, Ames, USA.

Jaselskis, E.J., Gao, Z., Welch, A. and O’Brien, D. (2003), "Pilot Study on Laser Scanning Technology for Transportation Projects", Proceedings the Mid-Continent Transportation Research Symposium, lowa State University, Ames, USA.

Jung, J., Hong, S., Jeong, S., Kim, S., Cho, H., Hong, S. and Heo, J. (2014), "Productive modeling for development of as-built BIM of existing indoor structures", Automation in Construction, 42, 68-77.

Kiziltas, S., Burcu, A., Ergen, E. and Pingbo, T. (2008), "Technological assessment and process implications of field data capture technologies for construction and facility/infrastructure management", ITcon, 13, 134-154.

Leung, T. and Malik, J. (2001), "Representing and Recognizing the Visual Appearance of Materials using Three-dimensional Textons", International Journal of Computer Vision, 43(1), 29-44.

Leutenegger, S., Chli, M. and Siegwart, R.Y. (2011), "BRISK: Binary Robust invariant scalable keypoints", Proceedings in Computer Vision (ICCV), 2548-2555.

Matas, J., Chum, O., Urban, M. and Pajdla, T. (2002), "Robust Wide Baseline Stereo from Maximally Stable Extremal Regions", The British Machine Vision Conference, BMVA Press, 384-393.

McArthur, J.J. (2015), “A Building Information Management (BIM) Framework and Supporting Case Study for Existing Building Operations, Maintenance and Sustainability", Procedia Engineering, 118, 1104-1111.

Mills, J., Andrews, D. and Barber, D. (2011), 3D Laser Scanning for Heritage, 2nd Edition, English Heritage Publishing, York, England.

Nasarudin, N.E.M. and Shafri, H. (2011), "Development and Utilization of Urban Spectral Library for Remote Sensing of Urban Environment", Journal of Urban and Environmental Engineering, 5(1), 45-56.

Olbrich, M., Graf, H., Kahn, S., Engelke, T., Keil, J., Riess, P., Webel, S., Bockholt, U., and Picinbono, G. (2013), "Augmented reality supporting user-centric building information management", The Visual Computer, 29(10), 1093-1105.

Rusinkiewicz, S. and Levoy, M. (2001), "Efficient variants of the ICP algorithm", Proceedings on 3-D Digital Imaging and Modeling, 145-152. 
Rusu, R.B. and Cousins, S. (2011), "3D is here: Point Cloud Library (PCL)”, International Conference on Robotics and Automation (ICRA), 1-4.

Rusu, R.B., Holzbach, A. Blodow, N. and Beetz, M. (2009), "Fast Geometric Point Labeling using Conditional Random Fields", International Conference on Intelligent Robots and Systems (IROS), 7-12.

Rusu, R.B., Marton, Z.C., Blodow, N., Dolha, M. and Beetz, M. (2008), "Towards 3D Point cloud based object maps for household environments", Robotics and Autonomous Systems, 56(11), 927-941.

Solihin, W. and Eastman, C. (2015), "Classification of rules for automated BIM rule checking development", Automation in Construction, 53, 69-82.

Son, H. and Kim, C. (2012), Automated Color Model-Based Concrete Detection in Construction-Site Images by Using Machine Learning Algorithms", Journal of Computing in Civil Engineering, 26(3), 421-433.

Steder, B., Rusu, R.B., Konolige, K. and Burgard, W. (2010), "NARF: 3D Range Image Features for Object Recognition", International Conference on Intelligent Robots and Systems (IROS), Taipei, Taiwan.

Tamas, L. and Kato, Z. (2013), "Targetless Calibration of a Lidar - Perspective Camera Pair", International Conference on Computer Vision Workshops (ICCVW), 668-675.

Tang, P., Huber, D., Akinci, B., Lipman, R. and Lytle, A. (2010), "Automatic reconstruction of as-built building information models from laser-scanned point clouds: A review of related techniques", Automation in Construction, 19(7), 829-843.

Teizer, J. (2015), "Status quo and open challenges in vision-based sensing and tracking of temporary resources on infrastructure construction sites", Advanced Engineering Informatics, 29(2), 225-238.

Tombari, F., Salti, S. and Di Stefano, L. (2012), "Performance Evaluation of 3D Keypoint Detectors", International Journal of Computer Vision, 102(1), 198-220.

Volk, R., Stengel, J. and Schultmann, F. (2014), "Building Information Modeling (BIM) for existing buildings - Literature review and future needs", Automation in Construction, 38,109-127.

Yalcinkaya, M. and Singh, V. (2015), "Patterns and trends in Building Information Modeling (BIM) research: A Latent Semantic Analysis", Automation in Construction, 59, 68-80.

Zhang, Z. (2000), "A flexible new technique for camera calibration", IEEE Transactions on Pattern Analysis and Machine Intelligence, 22(11), 1330-1334.

Zhu, Z. and Brilakis, I. (2009), "Comparison of Optical Sensor-Based Spatial Data Collection Techniques for Civil Infrastructure Modeling", Journal of Computing in Civil Engineering, 23(3), 170-177. 
(a)

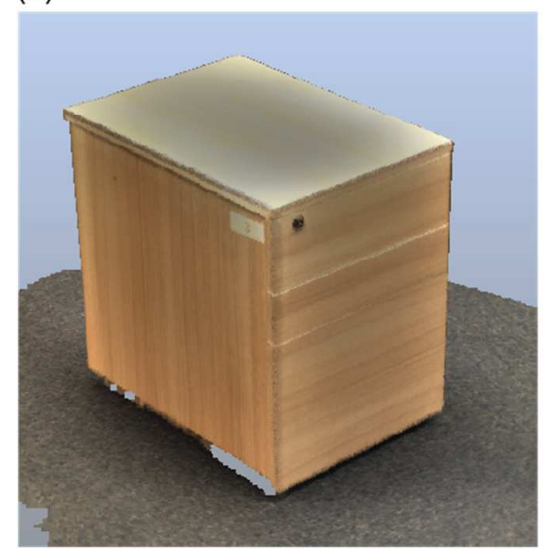

(b)

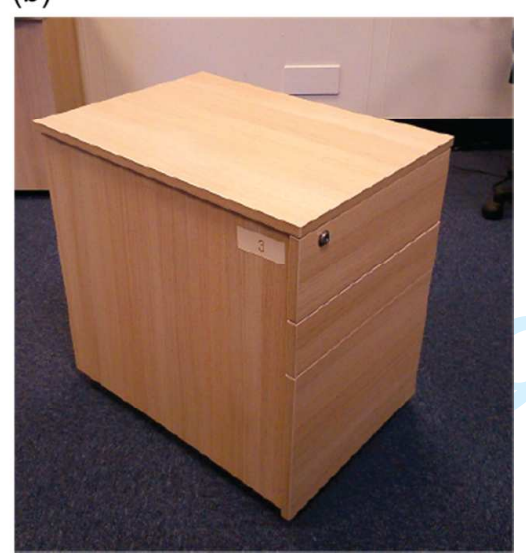

Figure 2. Examples of misrepresentation of colour appearance by a RGB camera. An office furniture unit was imaged by (a) the built-in RGB camera in the laser scanner and by (b) an external conventional RGB camera.

Information integration e.g. colour, spectral information

\section{As-build BIM}

Figure 1. Protocol of point cloud processing in a flow chart. 
(a)

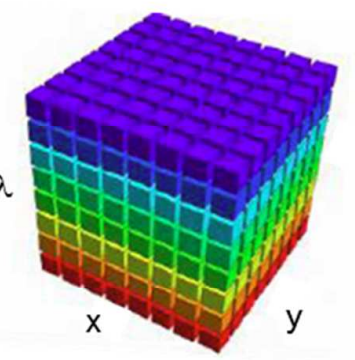

(b)

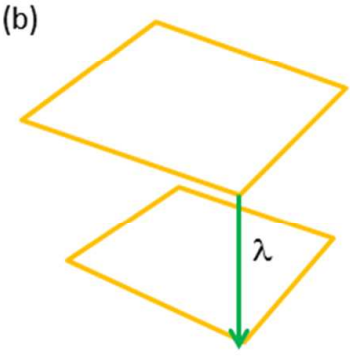

(c)

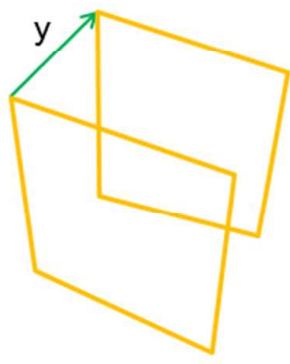

Figure 3. Schematic diagram explaining the classification of hyperspectral imaging systems. (a) the concept of the hyperspectral imaging can be explained by the hyperspectral-cube, (b) Spectral scan system with a tuneable spectral filter. (c) Line scan system 
(a)

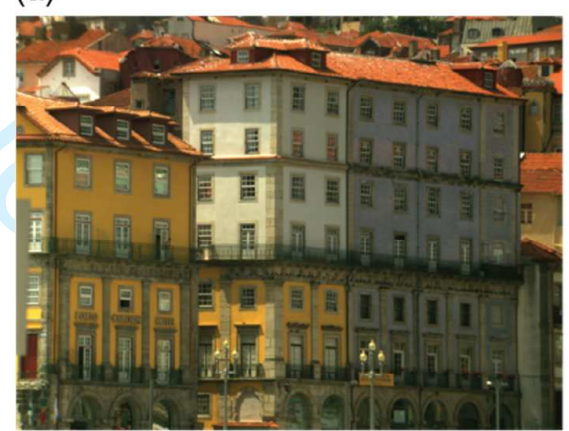

(c)

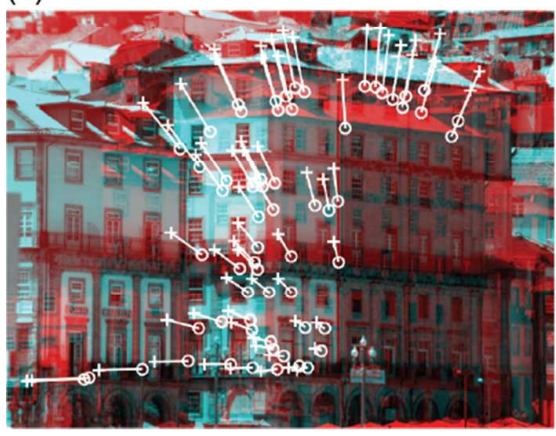

(b)

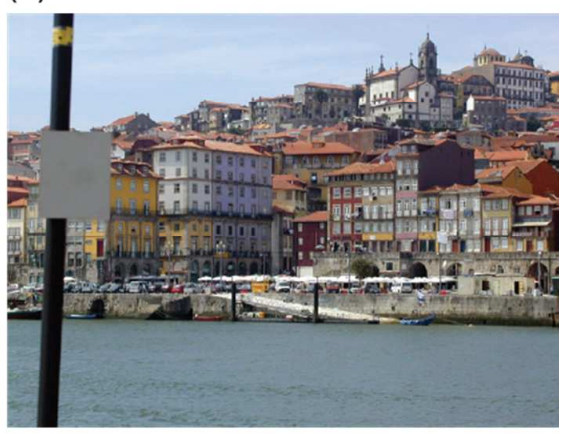

(d)

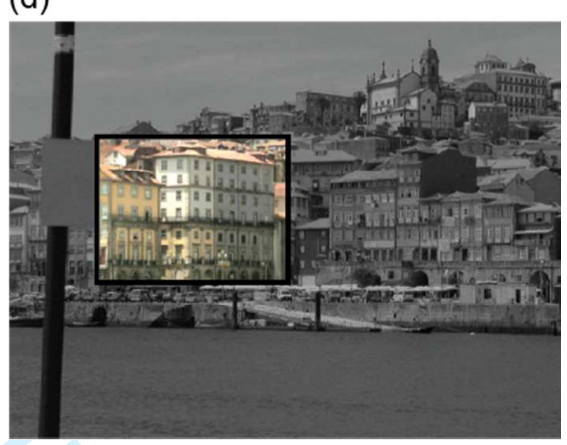

Figure 4. An demonstration image registration. A pair of 2D images acquired by (a) Colour image rendering of the hyperspectral data and (b) the same scene with a conventional RGB camera. (c) Detection of the SURF generated image key features. Circles and crosses are the features from each of the images in (a) and (b), coloured in blue (a) and red (b)for visibility. (d) shows the image registration. (For clarity a black frame has been inserted and the background has been converted to grey scale). 